\title{
PlantMolecularTasteDB: A Database of Taste Active Phytochemicals
}

\author{
Teodora-Cristiana Gradinaru ${ }^{1 \dagger}$, Madalina Petran ${ }^{1 \dagger}$, Dorin Dragos ${ }^{2,3 * t}$ and Marilena Gilca ${ }^{1}$ \\ ${ }^{1}$ Department of Functional Sciences I/Biochemistry, Faculty of Medicine, Carol Davila University of Medicine and Pharmacy, \\ Bucharest, Romania, ${ }^{2}$ Department of Medical Semiology, Faculty of Medicine, Carol Davila University of Medicine and Pharmacy, \\ Bucharest, Romania, ${ }^{3}$ 1st Internal Medicine Clinic, University Emergency Hospital Bucharest, Carol Davila University of Medicine \\ and Pharmacy, Bucharest, Romania
}

Keywords: taste, bitter, sweet, sour, pungent, astringent, phytochemicals, antiinflammatory

\section{INTRODUCTION}

In traditional medicine taste of medicinal plants represents one of the ethnopharmacological descriptors used for selection of the optimal herbal treatment of various ailments (Brett and Heinrich, 1998; Leonti et al., 2002; Gollin, 2004; Gilca and Barbulescu, 2015). Accumulating scientific evidence indicates that this ancient vision on the intrinsic therapeutic potency of herbal taste may not be completely devoid of a biological foundation (Gollin, 2004; Gilca and Dragos, 2017). Recent discoveries in taste science lead to the astonishing conclusion that the whole human body is endowed with a diffuse chemosensory system consisting of taste receptors and other chemesthesis sensors (Sbarbati and Osculati, 2005; Behrens and Meyerhof, 2011; Laffitte et al., 2014). This widespread extrasensorial expression of taste receptors and other oral chemosensors is increasingly recognized as a molecular basis for their non-gustative roles in many important biological processes, such as digestion (Harada et al., 2019), immune response (Douglas and Cohen, 2017), inflammation (Sharma et al., 2017), cell differentiation (Masubuchi et al., 2013), regulation of endocrine secretion (Clark et al., 2015), and many others (Behrens and Meyerhof, 2011; Laffitte et al., 2014).

Scientists have already suggested that these taste receptors and chemosensors might be druggable, thus having therapeutic potential (Lee et al., 2019). For instance, bitter taste receptors are considered potential critical players and therapeutic targets in inflammatory obstructive lung disease (GrassinDelyle et al., 2019) and genito-urinary tract infections/inflammation (Welcome, 2020), while pungency chemosensor TRPA1 (transient receptor potential cation channel ankyrin 1) was proposed as a regulator of neurogenic inflammation (Logashina et al., 2019). This explains the recently renewed interest of scientific community for taste receptors and tastants.

Due to their great biodiversity, plants are a huge reservoir of taste active compounds characterized by an extreme chemical heterogeneity. More and more phytotastants and their biological roles mediated by taste receptors are discovered every year. Therefore, the development of a database focused on plant-derived tastants like PlantMolecularTasteDB valorizes this accumulating evidence and paves the way for a deeper understanding of the taste-related traditional medical epistemology.

PlantMolecularTasteDB is distinctive from other similar resources, such as BitterDB (Wiener et al., 2012; Dagan-Wiener et al., 2019) and SuperSweetDB (Ahmed et al., 2011), by focusing on the complex gustative profile of plant derived tastants/compounds (meaning combination of all five basic tastes and/or orosensations such as pungency, astringency, etc. characteristic for each given tastant/compound) and on their evidence-based anti-inflammatory activity (Table 1). Furthermore,

Citation:

Gradinaru T-C, Petran M,

Dragos D and Gilca M (2022) PlantMolecularTasteDB: A Database

of Taste Active Phytochemicals.

Front. Pharmacol. 12:751712.

doi: 10.3389/fphar.2021.751712

Abbreviations: ASIC, acid-sensing ion channels; PMT, Plant Molecular Taste; TASR, taste receptor; TRP, transient receptor potential; TRPA1, transient receptor potential cation channel ankyrin 1; TRPC, Transient receptor potential canonical; TRPM, transient receptor potential melastatin; TRPV, transient receptor potential vanilloid. 
TABLE 1 | Comparison between various databases available on tastants and novelty of PlantMolecularTasteDB.

\begin{tabular}{|c|c|c|c|c|}
\hline Feature & PlantMolecularTasteDB & $\begin{array}{l}\text { BitterDB (Wiener et al., } \\
\text { 2012) }\end{array}$ & $\begin{array}{l}\text { SuperSweetDB (Ahmed } \\
\text { et al., 2011) }\end{array}$ & FlavorDB (Garg et al., 2018) \\
\hline \multirow[t]{3}{*}{ Compounds } & $\begin{array}{l}\text { Bitter, sweet, sour, salty, umami, pungent and } \\
\text { astringent compounds }\end{array}$ & Only bitter compounds & Only sweet tastants & $\begin{array}{l}\text { No distinction between taste and odour } \\
\text { of the compounds }\end{array}$ \\
\hline & Complete taste profile for each phytochemical & & & \\
\hline & Focused on plant-derived tastants & $\begin{array}{l}\text { Both natural and } \\
\text { synthetic tastants }\end{array}$ & $\begin{array}{l}\text { Both natural and synthetic } \\
\text { tastants }\end{array}$ & $\begin{array}{l}\text { Both natural and synthetic flavor } \\
\text { molecules }\end{array}$ \\
\hline Chemical class & Chemical class specified & - & Chemical class specified & Functional groups provided \\
\hline $\begin{array}{l}\text { Biological } \\
\text { activity }\end{array}$ & Antiinflammatory activity & - & - & - \\
\hline
\end{tabular}

PlantMolecularTasteDB is significantly richer in phytotastants than BitterDB and SuperSweetDB. It integrates data about all types of orosensorially active phytochemicals (not being focused on a single taste or orosensation).

\section{DATABASE OVERVIEW}

PlantMolecularTasteDB currently contains 1,527 phytochemicals that were reported in the literature as bitter (1,114 entries), sweet (263 entries), sour (61 entries), salty (7 entries), umami (25 entries), pungent (224 entries) or astringent (189 entries).

For each phytocompound PlantMolecularTasteDB offers information regarding synonyms, identifiers in international databases (PubChem ID, FooDB ID, HMDB ID, ChEMBL ID), molecular formula, chemical class, chemical structure, quantitative (taste threshold, where available) or qualitative sensorial data (taste/ orosensorial profile, where available), affinity for taste receptors or chemosensors (both positive and negative evidence, threshold value, EC50, type of interaction-agonist/antagonist, activation/inhibition threshold value, where available), anti-inflammatory activity (both positive and negative evidence, where available), references with links for the phytochemical gustative properties and biological activity. Regarding the chemical classes, the best represented are the alkaloids (32\%) and the terpenoids (21\%).

The main categories of taste receptors and chemosensors from various species, targeted by the phytochemicals in PlantMolecularTasteDB are: a. bitter taste receptors (TAS2Rs); b. sweet taste receptor (TAS1R2/TAS1R3); c. umami taste receptor (TAS1R1/TAS1R3); d. potential candidates for sour taste receptors (ASICs, PKD2L1, mPKD2L1, KIR); e. chemosensors involved in pungency and other chemesthesis sensation (cooling, warmth, heat, irritation) (TRPVs); f. others (e.g., $\mathrm{K}^{+}$channels, $\mathrm{Na}^{+}$channels, GPR40, GPR120, GPR84).

PlantMolecularTasteDB was developed for researchers in the field of (ethno)pharmacology, taste sciences, nutrition. PlantMolecularTasteDB interface allows its users to perform simple or advanced searches and browsing the dataset. The phytochemicals can be searched by simple search options using the following criteria: name, identifiers, taste (or trigeminal orosensations), taste receptor (or chemosensor), chemical class, anti-inflammatory activity. The advanced search allows to retrieve phytochemicals by a combination of criteria (e.g., bitter AND astringent, sweet AND saponin, triterpene AND TAS2R14, astringent AND anti-inflammatory, TRPA1 AND antiinflammatory).

\section{MATERIALS AND METHODS}

\subsection{Data Aquisition}

Data were collected from literature and publicly available databases. To begin with, a list of phytotastants was created using BitterDB (Wiener et al., 2012), SuperSweetDB (Ahmed et al., 2011), PubChem (https://pubchem.ncbi.nlm.nih.gov/), FooDB (https://foodb.ca/), PhytoMolTasteDB (Dragos and Gilca, 2018a). To this list, new taste active phytochemicals were added. They were identified by performing multiple systematic searches in PubMed, ScienceDirect and Google Scholar with various combinations of keywords: "phytochemical" AND "taste", specific phytochemical name AND taste (e.g., "colubrine" AND "taste"), "phytochemical" AND ("bitter" OR "sweet" OR "sour" OR "salty" OR "astringent" OR "pungent" OR "umami"), specific phytochemical name AND specific taste (e.g., "colubrine" AND "bitter"), "phytochemical" AND specific taste receptor/chemosensor name (e.g., "phytochemical" AND "TAS2R"). To extract data of interest (e.g., sensorial description, taste thresholds) apart from electronic search, we have also performed intensive manual search, wherever required, from hundreds of publications.

We have included 730 bitter phytochemicals not yet included in BitterDB and 235 sweet phytochemicals not yet included in SuperSweetDB.

Regarding the data on taste, few of the main literature resources cited were: Bitterness in food and beverages by Rouseff (1990) (97 phytochemicals), Merck index (78 phytochemicals) (Smith et al., 2001), Food chemistry by Belitz et al. (2009) (48 phytochemicals).

Phytochemicals were included in the database if they fulfilled simultaneously two conditions: 1 ) they were reported as taste active (sensorially or experimentally through ligand -taste receptor affinity assays) or as capable to induce other orosensations, such as astringency or pungency; 2) their exact structures were available in PubChem, FooDB, HMDB, ChEMBL or at least in one scientific publication.

Phytochemical structures that were not found in PubChem, FooDB, HMDB, ChEMBL were drawn manually using MarvinSketch 21.7 ChemAxon, Inc. (https://chemaxon.com). Afterwards, canonical and isomeric SMILES not available in PubChem were generated using Chemicalize tool, ChemAxon (www.chemicalize.com).

We additionally introduced in our database few peculiar categories of taste inactive phytochemicals, such as bitter 
masking sakuranetin and jaceosidin (Fletcher et al., 2011; Roland et al., 2014), sweetness enhancer arabinogalactan (Kim and Kinghorn, 2002), few precursors or metabolites of phytotastants, e.g. arctigenin -not bitter (the aglycon of a bitter compound, arctiin) (Matsuo et al., 1972), S-1-propenylL-cysteine sulfoxide, S-methyl-L-cysteine sulfoxide (pungency precursors) (Sun Yoo and Pike, 1998).

An original feature of PlantMolecularTasteDB absent from other taste-focused databases consists of data on the evidence-based biological activity of the phytotastants. The first biological activity introduced in the present version of PlantMolecularTasteDB is the antiinflammatory activity. For this purpose, a systematic literature search was performed using the phrase: [specific phytochemical name] AND (antiinflammatory OR anti-inflammatory OR inflammation)," [e.g., azadirachtin AND (antiinflammatory OR anti-inflammatory OR inflammation)] in PubMed, Elsevier databases and Google Scholar. We aimed to collect at least two relevant studies (when available), regardless of experimental model (in vitro, animal study, clinical study), study design, language, year of publication or publication status. Standardized criteria were utilized for selection. Antiinflammatory activity was considered evidencebased if supported by at least one in vitro, animal or human study.

The references regarding the taste or the anti-inflammatory activity were categorized as "insufficient evidence" if we encountered one of the following situations:

1) Only one original old reference (dated before 1960) was available.

2) References derived from papers published in journals not included in PubMed, ScienceDirect or other prestigious international databases.

3) Only ambiguous results were available. For instance, for 4,2 ' dihydroxychalcone, Roland et al. (2013) found ambiguous results for activation of both TAS2R14 and TAS2R39, when receptor assay was performed.

4) Only metabolomic profiling studies as references, e.g. kaempferol 3-O-p-coumaroyl-dirhamnosyl-glucoside (Zhu et al., 2017)

"Contradictory evidence" label was used if different authors reported opposite results, e.g., isovitexin reported as bitter (Zhu et al., 2017), non-bitter (Stark et al., 2005), and tasteless (Olennikov et al., 2015); capric acid reported as antiinflammatory (Lee and Kang, 2017) and also as proinflammatory (Tanaka et al., 2001).

Another original feature is a tool generating the so-called "Plant Molecular Taste" (PMT), which represents the virtual taste profile resulting from the contribution of all major orosensorially active phytocompounds found in the respective medicinal plant (Dragos and Gilca, 2018b). PMT is not necessarily the perceived taste, due to several reasons, including the quantitative one: a given phytotastant may be present in a certain plant only in a tiny amount, not surpassing its taste detection threshold. PMT was suggested to be a better predictor than the phytochemical class for the ethnopharmacological activities of the medicinal plants (Dragos and Gilca, 2018b). By convention, PMT graphical representation in PlantMolecularTasteDB takes into account each taste and trigeminal orosensation contribution to the gustative profile, calculated as a percentage of the total number of phytotastants present in that plant (or in a specific combination of phytochemicals).

\subsection{Database Structure}

\subsubsection{Web Server}

PlantMolecularTasteDB was built as a relational database. It is hosted on a MariaDB type server version 5.5.47, protocol version 10. The website has a PHP server side. An Apache HTTP Server enables web access. The site is well adapted to all the most popular browsers, such as Chrome, Firefox, Opera, Edge, and Safari.

\subsubsection{Visualisation Tools}

Marvin Sketch 21.7 plugin (http://www.chemaxon.com) enables the visualization of the molecular structure. PlantMolecularTasteDB contains a $2 \mathrm{D}$ compound structure display for each compound which is generated by Marvin JS 5.3.8, 2010 and a 3D rotation tool powered by ChemAxon (http://www.chemaxon.com). The chemical structure of phytochemicals can be downloaded as png or jpg.

\section{EXAMPLES OF USE}

\subsection{Searching for Sesquiterpenoid Lactones Which are Agonists of hTAS2Rs 3, 4, 5, 9, 10, 14, 30, 39, 40}

These compounds are of research interest because both sesquiterpenoid lactones and agonists of the mentioned hTAS2Rs displayed anti-inflammatory potential in various experimental studies (Hall et al., 1979; Hohmann et al., 2016; Grassin-Delyle et al., 2019). The advanced search with the following keywords "sesquiterpenoid lactone" AND "[hTAS2R3] OR [hTAS2R4] OR [hTAS2R5] OR [hTAS2R9] OR [hTAS2R10] OR [hTAS2R14] OR [hTAS2R30] OR [hTAS2R39] OR [hTAS2R40]" yielded 9 compounds (3-betahydroxy dihydrocostunolide, 3-beta-hydroxypelenolide, absinthin, alpha-santonin, arglabin, artemorin, costunolide, parthenolide, picrotoxinin), while the more advanced search with the following keywords "sesquiterpenoid lactone" AND "[hTAS2R3] OR [TAS2R4] OR [TAS2R5] OR [TAS2R9] OR [hTAS2R10] OR [hTAS2R14] OR [TAS2R30] OR [TAS2R39] OR [TAS2R40]" AND “antiinflammatory" produced only 6 compounds (absinthin, alpha-santonin, arglabin, artemorin, costunolide, parthenolide). Therefore the 6 compounds may be investigated for the contribution of their TAS2R agonist quality to the anti-inflammatory activity, while the other three compounds (3-beta-hydroxy dihydrocostunolide, 3-beta-hydroxypelenolide, picrotoxinin) may be further investigated for a potential antiinflammatory activity (mediated or not by TAS2Rs).

\subsection{Searching for Selective or Common Agonists of Taste Receptors}

In order to find selective agonists, the user should use the function Search $\rightarrow$ Advanced $\rightarrow$ Receptors all, and input the receptor of interest in the Agonist box, while the rest of the receptors in the Negative evidence box. For instance, 16 types of hTAS2Rs were 
Resulf

The combination of phytochemicals includes pimpinellin + bergapten + isopimpinellin + iso-bergapten + sphondin + phellopterin + oroselol + chlorogenic acid $+p$-coumaric acid + apigenin + kaempferol + luteolin + quercetin + rutin + o-coumaric acid + ferulic acid + vanillin + caffeic acid + p-hydroxybenzoic acid + catechin + protocatechuic acid + gallic acid + alpha-thujene + alpha-pinene + camphene + thuja-2,4(10)-diene + beta-pinene + myrcene + p-cymene + limonene + (Z)-beta-ocimene $+(\mathrm{E})$-beta-ocimene + gammaterpinene + 3,4-dimethylstyrene + 6-camphenone + isopentyl 2-methyl butanoate + 2-methylbutyl 2-methylbutanoate + 2-methylbutyl isovalerate $+(\mathrm{E})$-pinocarveol +4 terpinenol + myrtenal + octyl acetate + hexyl 2-methyl butanoate + hexyl 3-methylbutanoate + dihydrolinalyl acetate + bornyl acetate +octyl isobutyrate + alpha-copaene + beta-bourbonene + beta-elemene + alpha-cedrene +2 -epi- $\beta$-funebrene + beta-caryophyllene $+(E)$-alpha-bergamotene $+(E)$-beta-farnesene + germacrene $D+$ ar-curcumene + alpha-selinene + isodaucene + beta-bisabolene + beta-sesquiphellandrene $+(E)$-gamma-bisabolene + caryophyllene oxide + salvial-4(14)-en-1-one + humulene epoxide II + beta-atlantol + occidentalol acetate + amorpha-4,9-dien-2-ol + 1-octanol
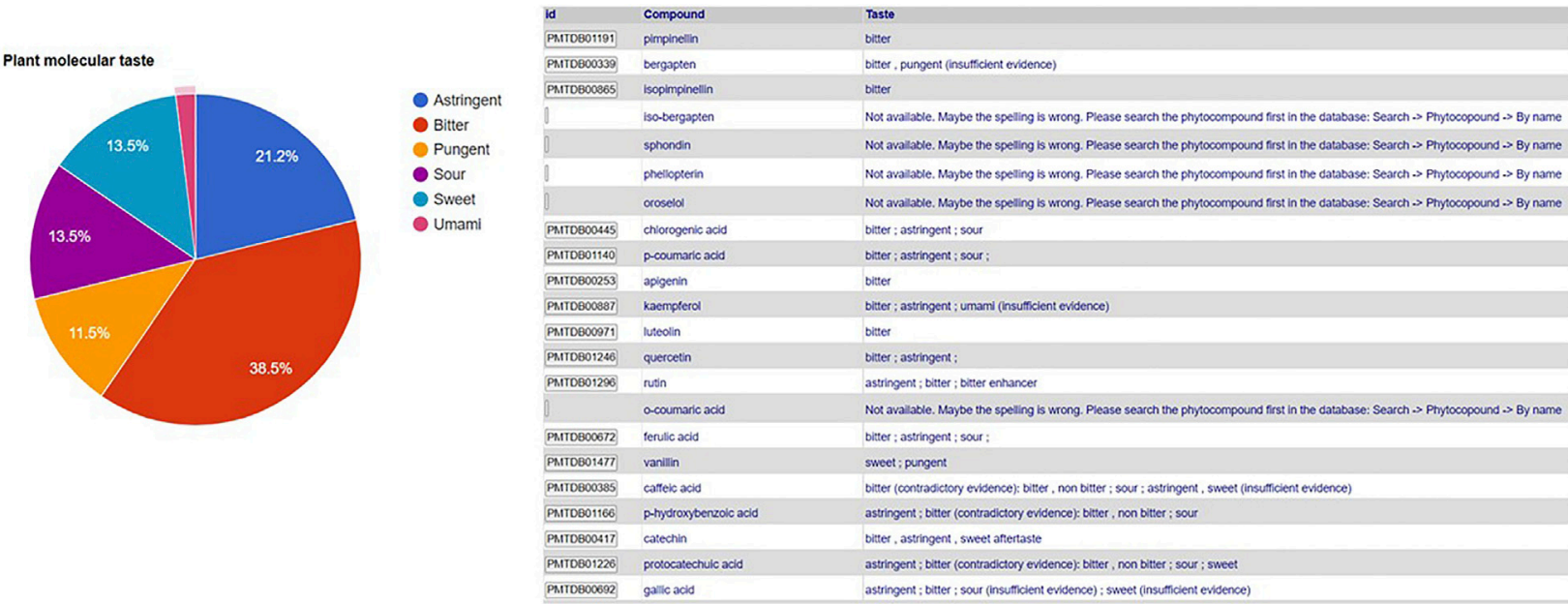

FIGURE 1 | Plant Molecular Taste of small hogweed (Heracleum sphondylium L., Apiaceae family) generated in PlantMolecularTasteDB.

found to be expressed in lung macrophages (Grassin-Delyle et al., 2015; Grassin-Delyle et al., 2019) and human bronchi (GrassinDelyle et al., 2013), being involved in bitter agonist dependentregulation of inflammation and bronchial relaxation. Therefore these TAS2Rs were proposed as new therapeutic targets in chronic obstructive lung diseases such as asthma (GrassinDelyle et al., 2013; Grassin-Delyle et al., 2015). In order to find selective agonists for instance for hTAS2R46, the user should perform the following search: Agonist $\rightarrow$ [hTAS2R46] and Negative evidence $\rightarrow$ " $\{$ [hTAS2R3] AND [hTAS2R4] AND [hTAS2R5] AND [hTAS2R7] AND [hTAS2R8] AND [hTAS2R9] AND [hTAS2R10] AND [hTAS2R14] AND [hTAS2R19] AND [hTAS2R20] AND [hTAS2R31] AND [hTAS2R38] AND [hTAS2R39] AND [hTAS2R43] AND [hTAS2R45]\}." This search leads to two selective agonists for hTAS2R46, which are not recognized by the rest of the receptors: dehydroandrographolide and oxymatrine. Selective agonists for each TAS2R are of interest in order to find the specific functional roles for each type of receptor.

Common agonists for at least 2 types of receptors may also be of interest in order to identify potential functional synergy between them, which may be used to increase the pharmacological efficacy of a ligand. For instance, hTAS2R5, hTAS2R10, and hTAS2R14 expressed in bronchi had a predominant role in bitter agonist-induced bronchial relaxation (Grassin-Delyle et al., 2013). A double search in PlantMoleculaTasteDB 1) Search $\rightarrow$ Receptor $\rightarrow$ “ $\{[$ hTAS2R5] AND [hTAS2R10] AND [hTAS2R14]\}"; 2) Search $\rightarrow$ Receptor $\rightarrow$ “\{[hTAS2R10] AND [hTAS2R14]\}"), found no common agonist for all the three receptors, but 22 common agonists for hTAS2R10 and hTAS2R14.

\subsection{Generating Plant Molecular Taste for a Medicinal Plant (Whole Composition or Partial Composition)}

In order to obtain a PMT, the user need to upload the composition of that plant (the names of constituent phytochemicals separated by semicolons in the text area at the end of the menu path Tools $\rightarrow$ Plant Molecular Taste $\rightarrow$ Select compounds from PlantMolecularTasteDB list OR Paste the list of compounds. This tool is especially of interest for medicinal plants insufficiently studied, since PMT may predict some of the plant (ethno)pharmacological activities that are worth studying experimentally (Gilca and Barbulescu, 2015; Dragos and Gilca, 2018a; Dragos and Gilca, 2018b). For instance, small hogweed (Heracleum sphondylium L., Apiaceae family) is traditionally used to treat several human ailments (e.g. flatulence, stomachache, diarrhea, epilepsy, hypertension, wounds, menstrual problems, impotence) (Bahadori et al., 2016), but is insufficiently studied. A search in PubMed with the keyword "Heracleum sphondylium" leads to only 21 papers, showing some evidence regarding only its antioxidant, antimutagenic, antimicrobial, and vasorelaxant activities. The phytochemical profile is relatively documented, the plant being rich in essential oil (Matejic et al., 2016), phenolic compounds (Uysal et al., 2019), and furanocoumarins (Dresler et al., 2018). The user has the possibility to upload: A) the entire list of the known major 
constituent phytochemicals of this plant (pimpinellin; bergapten; isopimpinellin etc. - see the box "Result" in Figure 1 for the complete list) or B) the composition of partial extracts (e.g., only essential oil constituents). The whole PMT is bitter $>$ astringent $>$ sweet $=$ sour $>$ pungent $>$ umami (Figure 1). The essential oil molecular taste is pungent $=$ bitter $>$ sweet. Interestingly, pungency, which is an organoleptic characteristic of essential oils in general, is traditionally associated with antiinfectious and antiparasitic activity, which were proven for Heracleum sphondylium essential oil by some experimental studies (Matejic et al., 2016; Uysal et al., 2019). Bitter taste was suggested as a predictor of antiinflammatory activity (Dragos and Gilca, 2018b; Dragos et al., 2021), while bitter taste receptors TAS2Rs showed recently anti-inflammatory effects (Sharma et al., 2017; Grassin-Delyle et al., 2019). The role of Heracleum sphondylium $\mathrm{L}$ as a source of inflammation modulating agents is yet to be explored.

\section{LIMITATIONS OF PLANTMOLECULARTASTEDB}

1) Not all the phytochemicals found in our database were investigated for their anti-inflammatory potential, therefore lack of any information marked by "No evidence" at the rubric Antiinflammatory activity means either negative evidence (true lack of anti-inflammatory activity) or lack of studies evaluating the anti-inflammatory activity of that compound.

2) Only a few physicochemical features (only molecular formula, chemical structure and chemical class) are available in the present version of PlantMolecularTasteDB.

3) The generation of PMT is dependent on the limited number of phytotastants existent in PlantMolecularTasteDB and on the scarcity of data regarding the chemical composition of certain medicinal plants.

4) Some of the phytochemicals submitted for generating PMT and declared "not found" may actually be present in PlantMolecularTasteDB but under a different name (orthographic variant or synonym). As a workaround for this issue the user might either choose from the list of phytocompounds (Tools $\rightarrow$ Plant Molecular Taste $\rightarrow$ Select compounds from PlantMolecularTasteDB) (which however does not include synonyms) or look for each phytochemical declared "not found," as the dedicated tool (Search $\rightarrow$ Phytocompound $\rightarrow$ By name) also explores the synonyms.

\section{REFERENCES}

Ahmed, J., Preissner, S., Dunkel, M., Worth, C. L., Eckert, A., and Preissner, R. (2011). SuperSweet--a Resource on Natural and Artificial Sweetening Agents. Nucleic Acids Res. 39, D377-D382. doi:10.1093/nar/gkq917

Bahadori, M. B., Dinparast, L., and Zengin, G. (2016). The Genus Heracleum: a Comprehensive Review on its Phytochemistry, Pharmacology, and Ethnobotanical Values as a Useful Herb. Compr. Rev. Food Sci. Food Saf. 15, 1018-1039. doi:10.1111/1541-4337.12222

Behrens, M., and Meyerhof, W. (2011). Gustatory and Extragustatory Functions of Mammalian Taste Receptors. Physiol. Behav. 105, 4-13. doi:10.1016/j.physbeh.2011.02.010

\section{CONCLUSION}

PlantMolecularTasteDB is the first database dedicated to all types of orosensorially active phytochemicals (bitter, sweet, sour, umami, salty, pungent, astringent phytochemicals). Its novelty over the other similar databases resides in the focus on the plant derived tastants, information related to sourness, pungency, astringency, complete taste profile, and antiinflammatory activity of the phytotastants. According to our knowledge, it is the database that contains the highest number of phytotastants and orosensation active phytochemicals. PlantMolecularTasteDB now provides a unique platform for further studies aiming to predict sensorial profile of unlisted phytocompounds or potential ligands for TASRs or TRPs. PlantMolecularTasteDB will be regularly upgraded with new phytotastants, physico-chemical features, biological activities, plants sources and interactive tools [e.g., taste predictive tool of newly discovered (phyto)chemicals].

\section{DATA AVAILABILITY STATEMENT}

PlantMolecularTasteDB is openly available at www. plantmoleculartastedb.org. Further inquiries can be directed to the corresponding author.

\section{AUTHOR CONTRIBUTIONS}

Conceptualization, MG and DD; methodology, DD and MG; software, DD; resources, MG; literature search T-CG, MP, and MG; data acquisition T-CG, MP, and DD; data curation, T-CG, $\mathrm{MP}, \mathrm{MG}$, and DD; writing-original draft preparation, $\mathrm{MG}, \mathrm{DD}$, T-CG, and MP; writing-review and editing, MG, DD, T-CG, and MP; visualization, DD; supervision MG and DD All authors have read and agreed to the present version of the manuscript.

\section{ACKNOWLEDGMENTS}

The authors obtained i-Depot certificate no. 122867/06-04-2020 (Benelux Office for Intellectual Property) for PlantMolecularTasteDB, available at www. plantmoleculartastedb.org. T-CG received one of the six Giract's European Flavor PhD Research Awards 2019-2020 for the best first year $\mathrm{PhD}$ project proposal.

Belitz, H. D., Grosch, W., and Schieberle, P. (2009). Food Chemistry. 4th ed. Leipzig: Springer Berlin Heidelberg. doi:10.1007/978-3-540-69934-7

Brett, J. A., and Heinrich, M. (1998). Culture, Perception and the Environment the Role of Chemosensory Perception. Angew. Bot. 72, 67-69.

Clark, A. A., Dotson, C. D., Elson, A. E., Voigt, A., Boehm, U., Meyerhof, W., et al. (2015). TAS2R Bitter Taste Receptors Regulate Thyroid Function. FASEB J. 29, 164-172. doi:10.1096/fj.14-262246

Dagan-Wiener, A., Di Pizio, A., Nissim, I., Bahia, M. S., Dubovski, N., Margulis, E., et al. (2019). BitterDB: Taste Ligands and Receptors Database in 2019. Nucleic Acids Res. 47, D1179-D1185. doi:10.1093/nar/gky974

Douglas, J. E., and Cohen, N. A. (2017). Taste Receptors Mediate Sinonasal Immunity and Respiratory Disease. Int. J. Mol. Sci. 18, 437. doi:10.3390/ijms18020437 
Dragos, D., and Gilca, M. (2018b). Taste of Phytocompounds: A Better Predictor for Ethnopharmacological Activities of Medicinal Plants Than the Phytochemical Class? J. Ethnopharmacol 220, 129-146. doi:10.1016/j.jep.2018.03.034

Dragos, D., and Gilca, M. (2018a). PhytoMolecularTasteDB: An Integrative Database on the "molecular Taste" of Indian Medicinal Plants. Data in Brief 19, 1237-1241. doi:10.1016/j.dib.2018.04.048

Dragos, D., Petran, M., Gradinaru, T., and Gilca, M. (2021). "P134. Do Bitter Phytocompounds Target Inflammation-Related Macromolecules," in The 20th International Congress of International Society for Ethnopharmacology, Virtual Congress, Greece, April 18th - 20th, 247.

Dresler, S., Bogucka-Kocka, A., Kováčik, J., Kubrak, T., Strzemski, M., WójciakKosior, M., et al. (2018). Separation and Determination of Coumarins Including Furanocoumarins Using Micellar Electrokinetic Capillary Chromatography. Talanta 187, 120-124. doi:10.1016/j.talanta.2018.05.024

Fletcher, J. N., Kinghorn, A. D., Slack, J. P., McCluskey, T. S., Odley, A., and Jia, Z. (2011). In Vitro Evaluation of Flavonoids from Eriodictyon Californicum for Antagonist Activity against the Bitterness Receptor hTAS2R31. J. Agric. Food Chem. 59, 13117-13121. doi:10.1021/jf204359q

Gilca, M., and Barbulescu, A. (2015). Taste of Medicinal Plants: a Potential Tool in Predicting Ethnopharmacological Activities? J. Ethnopharmacol. 174, 464-473. doi:10.1016/j.jep.2015.08.040

Gilca, M., and Dragos, D. (2017). Extraoral Taste Receptor Discovery: New Light on Ayurvedic Pharmacology. Evid. Based Complement. Alternat Med. 2017, 5435831-5435930. doi:10.1155/2017/5435831

Gollin, L. X. (2004). Subtle and Profound Sensory Attributes of Medicinal Plants Among the Kenyah Leppo' Ke of East Kalimantan, Borneo. J. Ethnobiol. 24, 173-201. Available at: http://direct.biostor.org/reference/175093.

Grassin-Delyle, S., Abrial, C., Fayad-Kobeissi, S., Brollo, M., Faisy, C., Alvarez, J. C., et al. (2013). The Expression and Relaxant Effect of Bitter Taste Receptors in Human Bronchi. Respir. Res. 14, 134. doi:10.1186/1465-9921-14-134

Grassin-Delyle, S., Naline, E., and Devillier, P. (2015). Taste Receptors in Asthma. Curr. Opin. Allergy Clin. Immunol. 15, 63-69. doi:10.1097/ACI.0000000000000137

Grassin-Delyle, S., Salvator, H., Mantov, N., Abrial, C., Brollo, M., Faisy, C., et al. (2019). Bitter Taste Receptors (TAS2Rs) in Human Lung Macrophages: Receptor Expression and Inhibitory Effects of TAS2R Agonists. Front. Physiol. 10, 1267. Available at: https://www.frontiersin.org/article/10.3389/ fphys.2019.01267. doi:10.3389/fphys.2019.0126710.3389/fphys.2019.01267

Hall, I. H., Lee, K. H., Starnes, C. O., Sumida, Y., Wu, R. Y., Waddell, T. G., et al. (1979). Anti-inflammatory Activity of Sesquiterpene Lactones and Related Compounds. J. Pharm. Sci. 68, 537-542. doi:10.1002/jps.2600680505

Harada, Y., Koseki, J., Sekine, H., Fujitsuka, N., and Kobayashi, H. (2019). Role of Bitter Taste Receptors in Regulating Gastric Accommodation in Guinea Pigs. J. Pharmacol. Exp. Ther. 369, 466-472. doi:10.1124/jpet.118.256008

Hohmann, M. S. N., Longhi-Balbinot, D. T., Guazelli, C. F. S., Navarro, S. A., Zarpelon, A. C., Casagrande, R., et al. (2016). "Sesquiterpene Lactones," in Studies in Natural Products Chemistry (Amsterdam, Boston, Heidelberg, London, New York, Oxford, Paris, San Diego, San Francisco, Singapore, Sydney, Tokyo: Elsevier), 243-264. doi:10.1016/b978-0-444-63601-0.00007-7

Kim, N.-C., and Kinghorn, A. D. (2002). "Sweet-tasting and Sweetness-Modifying Constituents of Plants. Studies in Natural Products Chemistry. 27, 3. doi:10.1016/S1572-5995(02)80033-3

Laffitte, A., Neiers, F., and Briand, L. (2014). Functional Roles of the Sweet Taste Receptor in Oral and Extraoral Tissues. Curr. Opin. Clin. Nutr. Metab. Care 17, 379-385. doi:10.1097/MCO.0000000000000058

Lee, S. I., and Kang, K. S. (2017). Function of Capric Acid in CyclophosphamideInduced Intestinal Inflammation, Oxidative Stress, and Barrier Function in Pigs. Sci. Rep. 7, 16530. doi:10.1038/s41598-017-16561-5

Lee, S. J., Depoortere, I., and Hatt, H. (2019). Therapeutic Potential of Ectopic Olfactory and Taste Receptors. Nat. Rev. Drug Discov. 18, 116-138. doi:10.1038/s41573-018-0002-3

Leonti, M., Sticher, O., and Heinrich, M. (2002). Medicinal Plants of the Popoluca, México: Organoleptic Properties as Indigenous Selection Criteria. J. Ethnopharmacol. 81, 307-315. doi:10.1016/S0378-8741(02)00078-8

Logashina, Y. A., Korolkova, Y. V., Kozlov, S. A., and Andreev, Y. A. (2019). TRPA1 Channel as a Regulator of Neurogenic Inflammation and Pain: Structure, Function, Role in Pathophysiology, and Therapeutic Potential of Ligands. Biochemistry (Mosc) 84, 101-118. doi:10.1134/S0006297919020020

Masubuchi, Y., Nakagawa, Y., Ma, J., Sasaki, T., Kitamura, T., Yamamoto, Y., et al. (2013). A Novel Regulatory Function of Sweet Taste-Sensing Receptor in
Adipogenic Differentiation of 3T3-L1 Cells. PLoS One 8, e54500. doi:10.1371/journal.pone.0054500

Matejic, J. S., Dzamic, A. M., Mihajilov-Krstev, T., Ristic, M. S., Randelovic, V. N., Krivošej, Z. Đ., et al. (2016). Chemical Composition, Antioxidant and Antimicrobial Properties of Essential Oil and Extracts fromHeracleum sphondyliumL. J. Essent. Oil Bearing Plants 19, 944-953. doi:10.1080/0972060X.2014.986538

Matsuo, K., Tokoroyama, T., and Kubota, T. (1972). Bitter Constituents of Forsythia Viridissima. Phytochemistry 11, 1522-1523. doi:10.1016/s0031-9422(00)90135-3 Olennikov, D. N., Kashchenko, N. I., Chirikova, N. K., Koryakina, L. P., and Vladimirov, L. N. (2015). Bitter Gentian Teas: Nutritional and Phytochemical Profiles, Polysaccharide Characterisation and Bioactivity. Molecules 20, 20014-20030. doi:10.3390/molecules201119674

Roland, W. S., Gouka, R. J., Gruppen, H., Driesse, M., van Buren, L., Smit, G., et al. (2014). 6-Methoxyflavanones as Bitter Taste Receptor Blockers for hTAS2R39. PLoS One 9, e94451. doi:10.1371/journal.pone.0094451

Roland, W. S., van Buren, L., Gruppen, H., Driesse, M., Gouka, R. J., Smit, G., et al. (2013). Bitter Taste Receptor Activation by Flavonoids and Isoflavonoids: Modeled Structural Requirements for Activation of hTAS2R14 and hTAS2R39. J. Agric. Food Chem. 61, 10454-10466. doi:10.1021/jf403387p

Rouseff, R. L. (1990). Bitterness in Foods and Beverages. Netherlands: Elsevier Amsterdam.

Sbarbati, A., and Osculati, F. (2005). The Taste Cell-Related Diffuse Chemosensory System. Prog. Neurobiol. 75, 295-307. doi:10.1016/j.pneurobio.2005.03.001

Sharma, P., Yi, R., Nayak, A. P., Wang, N., Tang, F., Knight, M. J., et al. (2017). Bitter Taste Receptor Agonists Mitigate Features of Allergic Asthma in Mice. Sci. Rep. 7, 46166. doi:10.1038/srep46166

Smith, A., Heckelman, P. E., Obenchain, J. R., Gallipeau, J. A. R., D’Arecca, M. A., and Budavari, S. (2001). The Merck Index. Thirteenth Edition. Whitehouse Station. New Jersey, USA: Merck Co., Inc.

Stark, T., Bareuther, S., and Hofmann, T. (2005). Sensory-Guided Decomposition of Roasted Cocoa Nibs (Theobroma Cacao) and Structure Determination of TasteActive Polyphenols. J. Agric. Food Chem. 53, 5407-5418. doi:10.1021/jf050457y

Sun Yoo, K., and Pike, L. M. (1998). Determination of flavor precursor compound s-alk(en)yl-L-cysteine sulfoxides by an HPLC method and their distribution in allium species. Scientia Horticulturae 75, 1-10. doi:10.1016/S0304-4238(98)00107-1

Tanaka, S., Saitoh, O., Tabata, K., Matsuse, R., Kojima, K., Sugi, K., et al. (2001). Medium-chain Fatty Acids Stimulate Interleukin-8 Production in Caco-2 Cells with Different Mechanisms from Long-Chain Fatty Acids. J. Gastroenterol. Hepatol. 16, 748-754. doi:10.1046/j.1440-1746.2001.02537.x

Uysal, A., Ozer, O. Y., Zengin, G., Stefanucci, A., Mollica, A., Picot-Allain, C. M. N., et al. (2019). Multifunctional Approaches to Provide Potential Pharmacophores for the Pharmacy Shelf: Heracleum Sphondylium L. Subsp. Ternatum (Velen.) Brummitt. Comput. Biol. Chem. 78, 64-73. doi:10.1016/j.compbiolchem.2018.11.018

Welcome, M. O. (2020). The Bitterness of Genitourinary Infections: Properties, Ligands of Genitourinary Bitter Taste Receptors and Mechanisms Linking Taste Sensing to Inflammatory Processes in the Genitourinary Tract. Eur. J. Obstet. Gynecol. Reprod. Biol. 247, 101-110. doi:10.1016/j.ejogrb.2020.02.015

Wiener, A., Shudler, M., Levit, A., and Niv, M. Y. (2012). BitterDB: a Database of Bitter Compounds. Nucleic Acids Res. 40, D413-D419. doi:10.1093/nar/gkr755

Zhu, M., Li, N., Zhao, M., Yu, W., and Wu, J. L. (2017). Metabolomic Profiling Delineate Taste Qualities of tea Leaf Pubescence. Food Res. Int. 94, 36-44. doi:10.1016/j.foodres.2017.01.026

Conflict of Interest: The authors declare that the research was conducted in the absence of any commercial or financial relationships that could be construed as a potential conflict of interest.

Publisher's Note: All claims expressed in this article are solely those of the authors and do not necessarily represent those of their affiliated organizations, or those of the publisher, the editors and the reviewers. Any product that may be evaluated in this article, or claim that may be made by its manufacturer, is not guaranteed or endorsed by the publisher.

Copyright (C) 2022 Gradinaru, Petran, Dragos and Gilca. This is an open-access article distributed under the terms of the Creative Commons Attribution License (CC BY). The use, distribution or reproduction in other forums is permitted, provided the original author(s) and the copyright owner(s) are credited and that the original publication in this journal is cited, in accordance with accepted academic practice. No use, distribution or reproduction is permitted which does not comply with these terms. 